\title{
Alfred Pikalo zum 70. Geburtstag
}

Alfred Pikalo, Notar und Honorarprofessor an der Faculté Internationale de Droit Rural et des Sciences Sociales Agraires de Montpellier, vollendete am 18. November 1977 sein 70. Lebensjahr. In Marchegg bei Wien geboren, verbrachte er bereits seine Jugend und überwiegend auch die späteren Jahre in Köln und im Rheinland. Auch außerhalb seiner speziellen Fachgebiete - dem Boden- und Umweltrecht und insbesondere dem Agrarrecht - ist er national und international als in der Praxis erfahrener Jurist und als Wissenschaftler bekannt und geschätzt. Auch diejenigen, die ihn näher kennen, sind sich gelegentlich nicht ganz einig, ob die Verknüpfung von juristischer Praxis und Wissenschaft Ausdruck seiner Person ist, die nach Gestaltung und Einflußnahme gleichermaßen wie nach wissenschaftlicher Durchdringung und vorwärts blickender Forschung gerichtet ist, oder ob gerade die Kombination von praktischer Tätigkeit und wissenschaftlicher Arbeit ihrerseits es ist, die die Persönlichkeit von Pikalo geformt hat. Jedenfalls ist gerade die in diesem $\mathrm{Maß}$ ebenso seltene wie insbesondere heute erforderliche - ja in seinen Rechtsgebieten unerläßliche - Verbindung von Praxis und Wissenschaft für ihn kennzeichnend.

Das Sammeln der praktischen Erfahrung begann Pikalo mit der nach dem Assessorexamen aufgenommenen Tätigkeit als Richter in Köln (1934-1937), danach als Notarvertreter im Rheinland, vor allem in Köln, und ab 1939 mit seiner Tätigkeit bei der Reichsnotarkammer in Berlin. 1942 wurde er Notar in Zülpich. Von 1943 bis 1945 war er zum Wehrdienst eingezogen. Nach Kriegsende setzte Pikalo seine Tätigkeit als Notar fort, seit 1952 bis heute amtiert er als Notar in Düren.

Die wissenschaftliche Betätigung begann Pikalo nach dem Studium der Rechtsund Staatswissenschaften an den Universitäten Köln und Berlin mit seiner Promotion 1930 an der Rechtswissenschaftlichen Fakultät in Köln. Nachdem er bereits zahlreiche juristische Arbeiten im In- und Ausland, insbesondere in Italien, Frankreich, Iberoamerika, veröffentlicht hatte, erhielt er 1960 einen Lehrauftrag an der Rechtswissenschaftlichen Fakultät der Universität zu Köln, den er bis 1968 wahrgenommen hat. In dieser Zeit betreute er mit besonderen Erfolgen mehr als 30 Doktoranden. Außerdem hielt er auf dem Gebiet des Bodenrechts Vorlesungen am Institut Universitaire International Luxembourgeois, Luxembourg. In Würdigung seiner wissenschaftlichen Verdienste wurde er 1971 zum Honorarprofessor an der Faculté Internationale de Droit Rural et des Sciences Sociales Agraires (Montpellier) ernannt. Von den vorangegangenen und nachfolgenden Arbeiten sind insbesondere hervorzuheben Werke wie ,Land- und forstwirtschaftliches Grundstücksverkehrs- und Erbrecht im westlichen Europa (1961), der - mit Bendel verfaßte - Kommentar „Grundstücksverkehrsgesetz" (1963) sowie der - mit Faßbender und Hötzel herausgegebene - ,Kommentar zur Höfeordnung" (1978). Scharfe Strukturanalysen und klare Funktionsbestimmun- 
gen waren in anderen Arbeiten vornehmlich der Eigentums- und Unternehmensverfassung und ihren Problemen gewidmet. Gespannt erwartet man seine jüngst noch übernommene Bearbeitung im ,Staudinger" zum neuen Landpachtrecht.

Manches von dem, was hier im Zusammenhang mit seiner praktischen und wissenschaftlichen Arbeit gesagt ist, kann nicht eigentlich dem einen oder dem anderen Wirkungsbereich allein zugeordnet werden, sondern dürfte eher als Ausdruck der eingangs angesprochenen ,Verbindung“ zu werten sein. Durch diese Kombination und ihre integrativen Bezüge sind gekennzeichnet beispielsweise seine Vorträge auf verschiedenen Europäischen Agrarrechtskongressen, auf Goslarer Agrarrechtsseminaren und am Institut des Hautes Etudes de Droit Rural et d'Economie Agricole, Paris. Nicht weniger gilt dies für seine Vortragstätigkeit an der Richterakademie in Schleswig-Holstein und bei der Rechts- und Staatswissenschaftlichen Gesellschaft zu Siegen. Am stärksten aber trifft dies zu für die seit der Gründung ausgeübte Tätigkeit als Präsident der Vereinigung für Deutsches und Europäisches Agrar- und Umweltrecht (V. A. U. R.) sowie für sein Wirken auf internationaler Ebene als Präsident des Comité Européen de Droit Rural (C. E. D. R.), Paris.

Unter der Präsidentschaft von Pikalo findet der X. Europäische Agrarrechtskongreß des C. E. D. R. in Berlin (1979) statt. Weiter ist hervorzuheben, daß Pikalo dem Vorstand des Düsseldorfer Josef-Humar-Instituts - Institut für Boden-, Bau-, Agrar- und Umweltrecht - angehört und zugleich die Funktion eines dortigen Institutsdirektors inne hat. In Anerkennung seiner Verdienste wurden ihm höchste Ehrungen und Ordensverleihungen, vorwiegend aus dem französischen, belgischen und spanischen Bereich, zuteil; in der Bundesrepublik Deutschland wurde ihm anläßlich seines 70 . Geburtstages die Franz-Böhm-Medaille verliehen. Alle diese Auszeichnungen bekunden die Resonanz eines sich immer reicher entfaltenden Werkes. Gleichermaßen bezeugen sie die Wirkung seiner wissenschaftlichen Forschungstätigkeit wie die als akademischer Lehrer, dem die Gabe des öffentlichen Wortes geschenkt und dessen Faszination die völlige Hingabe an den Vortrag ist; sie bekunden aber ebenso und vielleicht noch mehr die Zuneigung, die ihm seine persönlichen Eigenschaften in seinem weiten Wirken erworben haben. So steht vor uns ein imponierendes Lebenswerk. Es war nur möglich dank der lebendigen und fesselnden Persönlichkeit, die Schüler, Kollegen und Freunde immer wieder in ihren Bann gezogen hat. Nicht Sichten, Summieren und Abschließen ist Pikalos Wirken; sein Eidos ist Aufbruch und Erkundung. So ist zu hoffen, daß Alfred Pikalo, der sich unveränderter Schaffenskraft und Frische erfreut, weiterhin die Kraft behält, mit seiner Erfahrung und mit wissenschaftlichem Einsatz an der Ausformung der mit der Boden- und Umweltgestaltung in Beziehung stehenden Rechtsprobleme teilzunehmen und die Weiterentwicklung der der Gestaltung von Recht, Umwelt und Gesellschaft dienenden rechtsstaatlichen Prinzipien auf nationaler wie internationaler Ebene zu fördern.

Für die Kollegen, Freunde und Schüler: Günther Frohberg, Otto Kimminich, Robert Weimar 